\title{
Prevalencia de hipertensión arterial y factor es asociados en la población rural marginada
}

Jesús Fernando Guerrero-Romero, M.C.,1) Martha Rodríguez-Morán M.C., M. en C.(1)

\section{Guerrero-Romero JF, Rodríguez-Morán M. Prevalencia de hipertensión arterial y factores asociados en la población rural marginada.} Salud Publica Mex 1998;40:339-346.

\begin{abstract}
Resumen
Objetivo. D eterminar la prevalencia y los factores asociados a la hipertensión arterial sistémica (HAS) en la población rural marginada de Durango, México. Material y métodos. Se realizó un estudio transversal comparativo en 627 co munidades rurales, de las que aproximadamente $90 \%$ tiene 250 o menos habitantes. Se determinaron las cifras de presión arterial y las variables socio demo gráficas. Resultados. Se estudiaron 5802 sujetos, es decir, 4452 mujeres (76.7\%) y 1350 hombres (23.3\%). Se identificó HAS en 1271 individuos (21.9\%; IC 95\% 20.8-23.0), de los cuales 1011 eran mujeres (22.71\%; IC 95\% 21.5-23.9), y 260, hombres (19.26\%; IC $95 \%$ 17.2-21.4). Del total de la población blanco, 3018 personas (52.0\%) viven en comunidades con menos de 250 habitantes, 2080 (60.9\%) mujeres y 938 (31.1\%) hombres; en ésta se identificó HAS en 445 casos $(14.74 \%$; IC $95 \%$ 13.516.0), de los cuales 326 son mujeres (15.7\%; IC 95\% 14.1-17.3), y 119, hombres (12.7\%; IC $95 \%$ 10.6-14.9). Los principales factores de riesgo relacionados con la HAS fueron la obesidad, el diagnóstico de diabetes tipo 2 y el consumo de alco hol y tabaco. Conclusiones. Al parecer la prevalencia de HAS en la población rural marginada se relaciona con el grado de desarrollo de las comunidades.
\end{abstract}

Palabras clave: hipertensión/prevalencia; factores de riesgo; población rural; población marginada; México

\author{
Guerrero-Romero JF, Rodríguez-Morán M. \\ Prevalence and risk factors related \\ to systemic arterial hypertension \\ in a rural marginated population. \\ Salud Publica Mex 1998;40:339-346.
}

\begin{abstract}
A bstract
Objective. To determine the prevalence and risk factors related to systemic arterial hypertension $(\mathrm{SAH})$ in the rural marginated population of D urango, Mexico. Material and methods. A comparative cross-sectional study was performed in 627 rural communities, approximately $90 \%$ of which have 250 inhabitants or less. The arterial pressure and so ciodemographic variables were determined. Results. A total of 5802 subjects were studied, 4452 women (76.7\%) and 1350 men (23.3\%). SAH was fo und in 1271 individuals (21.9\%; Cl 95\% 20.8-23.0) of which 1011 were women $(22.71 \% ; \mathrm{Cl} 95 \%$ 21.5-23.9) and 260 were men (19.26\%; $\mathrm{Cl}$ $95 \%$ 17.2-21.4). Of the target population, 3018 individuals (52.0\%) live in communities of less than 250 inhabitants, 2 080 (60.9\%) women and 938 (31.1\%) men. In this group, SAH was identified in 445 cases $(14.74 \%$; $\mathrm{Cl}$ 95\% 13.5-16.0) of which 326 are women $(15.7 \% ; \mathrm{Cl} 95 \% 14.1-17.3)$ and 119 , men (12.7\%; $\mathrm{CI} 95 \%$ 10.6-14.9). The main risk factors related to $\mathrm{SAH}$ were obesity, type 2 diabetes, alcohol and tobacco consumption. Conclusions. The prevalence of SAH in the rural marginated population is apparently related to the degree of development of the communities.
\end{abstract}

Key words: hypertension/prevalence; risk factors; rural population; peripheric population; Mexico

(1) Instituto Mexicano del Seguro Social y Grupo de Investigación en Diabetes y Enfermedades Crónicas, D urango, México.

Fecha de recibido: 6 de mayo de 1997 - Fecha de aprobado: 25 de mayo de 1998

Solicitud de sobretiros: Dr. Jesús Fernando Guerrero Romero. Siqueiros 225, esq. c/C astañeda, zona centro, 34000 D urango, Durango, México. Correo electrónico: guerrero@ omanet.com.mx 
$\mathrm{E}$ n las últimas cinco décadas se han observado cambios en el panorama epidemiológico de México relacionados con la disminución de las enfermedades infecciosas, y que, combinados con otros cambios en el estilo de vida, han dado lugar al aumento progresivo de las enfermedades crónico-degenerativas, particularmente las cardiovasculares, ${ }^{1}$ de las cuales la hipertensión arterial es una de las más relevantes. ${ }^{2}$ En México se considera la presión arterial alta como un problema de salud pública, ${ }^{3,4}$ ya que favorece la aparición de otras enfermedades, ${ }^{1,2,5}$ acorta la vida de quien la padece $^{5}$ y se traduce en años potenciales de vida perdidos. ${ }^{2}$ En la población mexicana no se ha medido cabalmente la magnitud con que se presenta el problema de la hipertensión arterial; ${ }^{5}$ en ese contexto, se han realizado diversos estudios para establecer la prevalencia de la hipertensión arterial sistémica (PHAS) $)^{6-12}$ y se han notificado valores que fluctúan entre $10.2 \%{ }^{11}$ y $26.9 \% .^{12}$ En esas variaciones influyen de manera importante tanto los factores sociales y culturales ${ }^{7,8}$ inherentes a las poblaciones estudiadas, como las diferentes metodologías que se emplean y que impiden comparar los resultados y establecer con precisión la evolución de la hipertensión en México. La falta de conocimiento acerca de la epidemiología de las enfermedades crónicas dio lugar a la Encuesta Nacional de Enfermedades Crónicas (ENEC), que en 1991 notificó una PHAS de $24.6 \%{ }^{13}$ en México; dos años después, en 1993 , esa cifra se incrementó a $26.6 \%,{ }^{14}$ lo que refleja la importancia de esta enfermedad y su impacto en la salud pública. En las zonas rurales se han hecho menos esfuerzos para determinar la magnitud de este problema, si bien una gran parte de la población del país se concentra en el campo. Los estudios de hipertensión realizados en poblaciones rurales muestran una prevalencia que fluctúa entre $7.7^{15}$ y $10.0 \%$; estas variaciones dependen, además de la estructura de la población y de la metodología empleada, de las características de los sujetos de estudio, que en el área rural varían considerablemente de una región a otra. Ello motiva a la realización de estudios con métodos estandarizados que permitan comparar las características, los factores de riesgo predisponentes y la prevalencia de la hipertensión arterial en las diferentes áreas de la población rural del país.

\footnotetext{
* Saunders N. Trabajo presentado en el VIII Congreso Mundial de Cardiología 1978 sept; Tokio, Japón. Citado por: González-Caamaño A, Pérez-Balmes J, Nieto-Sánchez C, Vázquez-Contreras E. Importancia de las enfermedades cronicodegenerativas dentro del panorama epidemiológico actual de México. Salud Publica Mex $1986 ; 28: 8$.
}

En este trabajo se determinan la prevalencia y los factores asociados a la hipertensión arterial sistémica (HAS) en la población rural marginada atendida por el sistema IMSS-Solidaridad, en el estado de Durango, México.

\section{Material y métodos}

Como parte del estudio de prevalencia de diabetes mellitus no insulino-dependiente (DMNID) en la población rural marginada de Durango, México, ${ }^{16}$ que se desarrolló de enero de 1994 a noviembre de 1995, se realizó un estudio transversal comparativo para determinar la prevalencia de HAS en esa región que incluye 627 poblaciones, de las que aproximadamente $90 \%$ tienen menos de 250 habitantes. ${ }^{17}$ La población rural marginada, universo de este estudio, se ubica en el llamado "espinazo de la pobreza de México" y se caracteriza por vivir en condiciones de marginación media y de extrema pobreza. En este sector, 8 de cada 10 trabajadores se dedican a actividades ligadas a la tierra y practican una agricultura de temporal en un medio físico desfavorable; además, viven en zonas agrestes, mal comunicadas y en un marco de notables carencias respecto a los principales indicadores de vida: alimentación, escolaridad y estado físico; sus viviendas son de mala calidad, con una construcción rudimentaria y muy deteriorada, y carecen de agua potable, drenaje y energía eléctrica. ${ }^{17}$

El estudio se llevó a cabo en el marco de las actividades asistenciales del programa IMSS-Solidaridad, que atiende a la población rural marginada de Durango, constituida por 254617 habitantes, cifra equivalente a $25.88 \%$ de la población total del estado. Los médicos que laboran en ese programa se encargaron de realizar las mediciones.

La estandarización del procedimiento se realizó con base en un adiestramiento previo orientado a la unificación de criterios para evaluar la presión arterial (PA), así como para el manejo de las técnicas de entrevista y de la encuesta. El método uniforme para medir la PA fue el auscultatorio indirecto, utilizando esfigmomanómetros de mercurio, con redondeo de las cifras de PA a $2 \mathrm{mmHg}$. La columna de mercurio y la escala de lectura se colocaron en posición vertical, con la tubería de hule libre de compresiones o dobleces; antes de iniciar la lectura de la PA, el encargado se cercioraba de que el nivel inicial de la columna de mercurio se encontrara a $0 \mathrm{mmHg}$. El extremo distal del manguito colocado en el brazo debía estar a la altura del apéndice xifoides, con el brazo separado del cuerpo y ligeramente flexionado, con la cara palmar de la mano hacia arriba. El registro de la PA se basó en la 
percepción del latido braquial en el pliegue del codo. Primero se palpó el latido arterial braquial para colocar adecuadamente el estetoscopio en ese sitio. Para medir la presión auscultatoria se ajustó la velocidad de deflación para que cada ruido coincidiera con las líneas de la escala de la columna de mercurio. Se consideró como presión arterial sistólica y diastólica al registro del primero y quinto ruido de Korotkoff, respectivamente. La cifra que se tomó en cuenta fue el promedio de dos lecturas consecutivas de la PA, con cinco minutos de intervalo entre ambas. La PA se midió en el brazo izquierdo de los sujetos en reposo, sentados cómodamente y tan relajados como fuera posible. Al medir la PA con este procedimiento estandarizado, la variabilidad en las lecturas disminuyó, por lo que la validez interna del estudio incrementó.

Personal del programa IMSS-Solidaridad que visita con regularidad a los habitantes de las poblaciones blanco, les informó acerca de este estudio.

Esta investigación incluyó las 627 comunidades que constituyen el universo atendido por el programa IMSS-Solidaridad en Durango, México; en las comunidades con menos de 40 casas se hicieron visitas casa por casa; en las poblaciones con más de 40 casas, se procedió a tomar bloques de 10 y, por asignación aleatoria, se seleccionaron cinco casas por cada bloque, donde se entrevistó a los habitantes de 20 o más años de edad, que constituyen cerca de $48.64 \%$ (123 845 habitantes) de la población de la región en estudio. Cada comunidad se visitó por lo menos en cuatro ocasiones, y a los participantes en el estudio se les midió la PA por lo menos en dos ocasiones. Con esta técnica de muestreo se logró una mayor cobertura y participación de la población blanco.

El tamaño de la muestra se determinó con el programa estadístico True Epistat, tomando como parámetros la prevalencia de HAS en la región norte de México $(25 \%)$ y que notificó la ENEC, ${ }^{13}$ así como los valores de $\alpha$ y $\beta$ de 0.01 y 0.10 , respectivamente; el tamaño de muestra requerido fue de 4975 sujetos.

Se estableció el diagnóstico de HAS de acuerdo con los criterios del Joint National Committee on Detection, Evaluation and Treatment of High Blood Pressure, $^{7}$ que define la HAS por cifras iguales o mayores a 140/90 mmHg para la presión sistólica (PS) y diastólica (PD), respectivamente, determinadas por lo menos en dos ocasiones. Asimismo se consideró como hipertensos a los individuos con diagnóstico previo de HAS que recibían medicación antihipertensiva, independientemente de sus cifras tensionales.

Se determinaron la edad, el género, el salario, los antecedentes familiares de diabetes tipo 2 y de HAS, los antecedentes personales de diabetes y la historia de tabaquismo y consumo de alcohol.

Se consideró el antecedente familiar de diabetes y/o HAS ante la existencia de, por lo menos, un familiar en primer grado (padres o hermanos) que padeciera una o ambas enfermedades -diagnosticadas por un médico-y que recibieran tratamiento. Se consideró que había antecedente personal de diabetes cuando existía un diagnóstico previo de diabetes mellitus tipo 2 y el paciente se encontraba bajo tratamiento médico para control del padecimiento.

La historia de tabaquismo se estableció con base en el número de cigarrillos consumidos al día y la duración del hábito; de esta forma, se construyó el indicador cigarrillos/día.

Considerando la frecuencia, la intensidad y la duración del consumo de la bebida alcohólica de preferencia, así como el contenido de alcohol de la misma, se constituyó el indicador gramos de alcohol/día; se consideró que había alcoholismo leve cuando se consumían de 1 a $49 \mathrm{~g}$ alcohol/día; moderado, cuando la ingesta era de 50 a $99 \mathrm{~g}$ alcohol/día, y grave, cuando había un consumo de más de $100 \mathrm{~g}$ alcohol/día.

El peso se midió en una balanza tipo romano, que se calibró en forma previa a cada medición, con el sujeto sin calzado y en bipedestación; la talla se determinó con un estadímetro de Harpenden. Con base en las mediciones anteriores se estableció el índice de masa corporal (IMC) expresado como la relación $\mathrm{kg} / \mathrm{m}^{2}, \mathrm{y}$ se consideró como obesidad ${ }^{14}$ el IMC $\geq 30 \mathrm{~kg} / \mathrm{m}^{2}$.

\section{A nálisis estadístico}

Se recurrió a la estadística descriptiva, es decir, a medidas de tendencia central (media), de dispersión (desviación estándar) y porcentajes. En el caso de las variables dicotómicas (estimación de la prevalencia) se calculó el límite de confianza para la distribución binomial. La significancia de las diferencias entre los grupos se calculó con la prueba $\chi^{2}$, en el caso de las variables nominales y (previo análisis de la distribución muestral) con la prueba $t$ de Student (U de Mann-Whitney) para las variables continuas. Las diferencias entre los grupos con y sin HAS, distribuidos por género, se estimaron con análisis de varianza (ANOVA) de dos vías, para las variables númericas, y con $\chi^{2}$ de MantelHaenszel en el caso de las nominales. Para estimar la asociación de los factores de riesgo con la HAS, se realizó un análisis multivariado ajustado por edad y género, en el que se consideraron como variables independientes aquellas que mostraron asociación significativa en el análisis bivariado. 
La información se procesó mediante el paquete estadístico True Epistat. En todos los casos se consideró un intervalo de confianza (IC) de 95\%.

\section{Resultados}

Se integraron al estudio 5802 sujetos, de los cuales 4452 eran mujeres $(76.7 \%)$, y 1350 , hombres (23.3\%). Se identificó HAS en 1271 individuos (prevalencia 21.9\%, IC95\% 20.8-23.0). De los sujetos con hipertensión 1011 eran mujeres $(22.71 \%$, IC95\% 21.5-23.9), y 260, hombres (19.26\%, IC95\% 17.2-21.4); la distribución de HAS por género mostró una razón mujer/hombre de 1.18:1.

Del total de la población blanco, $3018(52.0 \%)$ sujetos vivían en comunidades de menos de 250 habitantes, de los cuales 2080 (60.9\%) eran mujeres, y 938 $(31.1 \%)$, hombres; en ésta se identificó HAS en 445 casos (14.74\%, IC95\% 13.5-16.0), esto es, en 326 mujeres (15.7\%, IC95\% 14.1-17.3) y en 119 hombres (12.7\%, IC95\% 10.6-14.9), para una razón mujer/hombre de 1.23:1.

De la población total de hipertensos, 879 (69.15\%) individuos tenían diagnóstico establecido en forma previa y $392(30.8 \%)$ fueron hallazgo de la encuesta, distribución que indica una razón hallazgo/diagnóstico previa de 0.44 . Entre las mujeres predominaron los casos de hipertensión con diagnóstico previo, y entre los hombres, los hallazgos de nuevo diagnóstico, en prácticamente todos los estratos de edad (cuadro I).
La presencia de HAS es relativamente baja hasta la edad de 34 años; a partir del grupo de 35 años se incrementa significativamente, de tal modo que la prevalencia más elevada se documenta en los sujetos de 65 a 74 años y en los mayores de 80 (cuadro I).

En el cuadro II se muestran las características generales de la población estudiada; en los pacientes con HAS se hacen evidentes factores como la edad, la obesidad y un mayor consumo de tabaco y de alcohol respecto a los sujetos sin hipertensión.

Se identificaron antecedentes familiares de HAS en 1994 (34.3\%) sujetos, de los cuales 626 (49.2\%) pertenecían al grupo con hipertensión, y $1368(23.6 \%)$, al que no tenía $(p<0.00001)$; de los casos con antecedente familiar positivo, 86.2\% (1719/1 994) correspondieron a la rama materna, y de ellos 527 presentaban hipertensión $(p<0.00001)$.

En el cuadro III se muestra la distribución por género de los principales factores asociados a la HAS; en los hombres con hipertensión predominó una mayor frecuencia de diabetes tipo 2 y de antecedentes familiares de hipertensión arterial pero, sobre todo, el elevado consumo de tabaco y alcohol, que se encontró en aproximadamente $99 \%$ de la población masculina con HAS. Por otro lado, tanto en las mujeres como en los hombres con hipertensión existe una mayor proporción de sujetos obesos y mayores de 55 años, respecto al grupo control. En el análisis multivariado, ajustado por edad y género, mostraron asociación con

\section{Cuadro I}

DiSTRIBUCIÓN, POR GÉNERO Y EDAD, DE LOS SUJETOS CON HIPERTENSIÓN ARTERIAL EN LA POBLACIÓN RURAL. Durango, México, 1994-1995

\begin{tabular}{|c|c|c|c|c|c|c|c|c|c|c|}
\hline \multirow{2}{*}{$\begin{array}{c}\text { Edad } \\
\text { (años) }\end{array}$} & \multirow[t]{2}{*}{$n$} & \multirow[t]{2}{*}{$\%$} & \multicolumn{2}{|c|}{ Mujeres \% } & \multirow{2}{*}{$\begin{array}{c}\text { Total } \\
\%\end{array}$} & \multirow[t]{2}{*}{$n$} & \multirow[t]{2}{*}{$\%$} & \multicolumn{2}{|c|}{ Hombres \% } & \multirow{2}{*}{$\begin{array}{r}\text { Tota } \\
\%\end{array}$} \\
\hline & & & Diagnóstico previo & Hallazgo & & & & Diagnóstico previo & Hallazgo & \\
\hline $20-24$ & 203 & 4.56 & 5.9 & 1.9 & 7.8 & 25 & 1.86 & 4.0 & 0.0 & 4.0 \\
\hline $25-29$ & 747 & 16.78 & 3.2 & 3.5 & 6.7 & 156 & 11.36 & 1.3 & 5.8 & 7.1 \\
\hline $30-34$ & 646 & 14.51 & 5.5 & 4.6 & 10.1 & 141 & 10.45 & 2.1 & 5.7 & 7.8 \\
\hline $35-39$ & 491 & 11.03 & 9.6 & 5.3 & 14.9 & 109 & 8.07 & 4.6 & 6.4 & 11.0 \\
\hline $40-44$ & 524 & 11.77 & 17.2 & 5.9 & 23.1 & 120 & 8.89 & 5.0 & 5.8 & 10.8 \\
\hline $45-49$ & 444 & 9.97 & 22.3 & 5.8 & 28.1 & 124 & 9.19 & 11.3 & 8.9 & 20.2 \\
\hline $50-54$ & 396 & 8.89 & 21.5 & 5.5 & 27.0 & 136 & 10.07 & 16.2 & 9.6 & 25.8 \\
\hline $55-59$ & 336 & 7.55 & 31.5 & 10.4 & 41.9 & 116 & 8.59 & 8.6 & 14.6 & 23.2 \\
\hline $60-64$ & 308 & 6.92 & 28.9 & 8.4 & 37.3 & 163 & 12.07 & 17.2 & 6.1 & 23.3 \\
\hline $65-69$ & 157 & 3.53 & 39.5 & 9.5 & 49.0 & 96 & 7.11 & 20.8 & 14.6 & 35.4 \\
\hline $70-74$ & 102 & 2.29 & 32.3 & 9.8 & 42.1 & 81 & 6.0 & 17.3 & 18.5 & 35.8 \\
\hline $75-79$ & 50 & 1.12 & 44.0 & 4.0 & 48.0 & 40 & 2.96 & 15.0 & 7.5 & 22.5 \\
\hline $80-84$ & 29 & 0.65 & 24.1 & 10.3 & 34.4 & 27 & 2.0 & 25.9 & 11.1 & 37.0 \\
\hline$\geq 85$ & 19 & 0.43 & 47.3 & 5.3 & 52.6 & 16 & 1.18 & 18.7 & 12.5 & 31.2 \\
\hline Total & 4452 & 100 & & & & 1350 & 100 & & & \\
\hline
\end{tabular}




\section{Características de la población rural. Durango, México, 1994-1995}

\begin{tabular}{|c|c|c|c|c|c|}
\hline & \multicolumn{2}{|c|}{ Con HAS } & \multicolumn{2}{|c|}{ Sin HAS } & \multirow[t]{2}{*}{ Valor de $p^{*}$} \\
\hline & $\begin{array}{c}\text { M ujeres } \\
=1011\end{array}$ & $\frac{\text { Hombres }}{n=260}$ & $\frac{M \text { ujeres }}{n=3441}$ & $\frac{\text { Hombres }}{n=1090}$ & \\
\hline Edad (años) & $51.5 \pm 13.8$ & $52.8 \pm 14.7$ & $40.1 \pm 13.4$ & $47.5 \pm 15.1$ & 0.0001 \\
\hline Indice de masa corporal $\left(\mathrm{kg} / \mathrm{m}^{2}\right)$ & $27.6 \pm 9.8$ & $28.2 \pm 9.1$ & $26.3 \pm 6.1$ & $24.8 \pm 4.8$ & 0.01 \\
\hline Tabaquismo (cigarros/día) ${ }^{\ddagger}$ & $18.1 \pm 3.2$ & $19.8 \pm 5.7$ & $9.1 \pm 2.0$ & $13.5 \pm 3.6$ & $<0.0001$ \\
\hline Consumo de alcohol (g alcohol/día) & $25.0 \pm 1.6$ & $75.0 \pm 2.0$ & $15.2 \pm 1.6$ & $22.1 \pm 1.5$ & $<0.0001$ \\
\hline
\end{tabular}

HAS: hipertensión arterial sistémica

* ANOVA de dos vías

‡ Expresa el consumo promedio de cigarros/día en los sujetos con hábito tabáquico

\section{Cuadro III}

\section{Factores asociados a hipertensión arterial en la población ruRal. Durango, México, 1994-1995}

\begin{tabular}{|c|c|c|c|c|c|c|c|c|c|}
\hline & \multicolumn{4}{|c|}{ Con HAS } & \multicolumn{4}{|c|}{$\mathrm{Sin} \mathrm{HAS}$} & \multirow[t]{2}{*}{ Valor de $p^{*}$} \\
\hline & \multicolumn{2}{|c|}{ Mujeres } & \multicolumn{2}{|c|}{ Hombres } & \multicolumn{2}{|c|}{ Mujeres } & $\begin{array}{r}\text { Hom } \\
n=1090\end{array}$ & $\frac{\mathrm{es}}{\%}$ & \\
\hline Edad $\geq 55$ años & 602 & 59.5 & 207 & 79.6 & 533 & 15.5 & 345 & 31.6 & $<0.0001$ \\
\hline$\left.\overline{0 \text { besidad }(I M C} \geq 30 \mathrm{~kg} / \mathrm{m}^{2}\right)$ & 240 & 23.4 & 80 & 30.8 & 145 & 4.2 & 79 & 7.2 & $<0.0001$ \\
\hline \multicolumn{10}{|l|}{ Ant. familiares de: } \\
\hline Hipertensión arterial & 444 & 43.9 & 182 & 70.0 & 1082 & 31.4 & 286 & 26.2 & $<0.0001$ \\
\hline Diabetes tipo 2 & 352 & 34.8 & 153 & 58.9 & 947 & 27.5 & 306 & 28.0 & $<0.0001$ \\
\hline Diabetes tipo 2 & 211 & 20.9 & 171 & 65.8 & 278 & 8.1 & 107 & 9.8 & $<0.0001$ \\
\hline Tabaquismo & 425 & 42.0 & 259 & 99.6 & 81 & 2.3 & 102 & 9.3 & $<0.0001$ \\
\hline \multicolumn{10}{|l|}{ Consumo de alcohol } \\
\hline Total & 191 & 18.9 & 257 & 98.8 & 122 & 3.5 & 385 & 35.3 & $<0.0001$ \\
\hline Leve (1 - 49 g/día) & 82 & 8.1 & 4 & 1.5 & 87 & 2.5 & 83 & 7.6 & \\
\hline Moderado (50 - 99 g/día) & 91 & 9.0 & 71 & 27.3 & 21 & 0.6 & 127 & 11.7 & \\
\hline Grave ( $\geq 100 \mathrm{~g} / \mathrm{d}$ ía) & 18 & 1.8 & 182 & 70.0 & 14 & 0.4 & 175 & 16.0 & \\
\hline
\end{tabular}

la HAS: la obesidad (razón de momios-RM-3.4, IC95\% $1.8-5.7, p=0.01)$; la diabetes tipo 2 (RM 4.2, IC95\% 2.36.4, $p=0.001$ ); el tabaquismo (RM 2.9; IC95\% 1.4-7.3, $p=0.01)$, y el consumo de alcohol $>50 \mathrm{~g} /$ día (RM 1.7; IC95\% 1.2-8.7, $p=0.04$ ).

\section{Discusión}

La hipertensión arterial se sitúa entre las enfermedades de mayor relevancia debido a su gran magnitud y amplia distribución, por lo que los esfuerzos dirigidos a determinar su prevalencia e identificar los factores de riesgo relacionados constituyen acciones prioritarias de salud pública, ${ }^{18}$ indispensables para establecer estrategias tendentes a disminuir la incidencia y a limitar la morbilidad relacionada con dicho padecimiento. ${ }^{1}$

En las poblaciones urbanas del norte del país la prevalencia de hipertensión es de $27.9 \%$; $^{14}$ en este estudio representó $21.9 \%$ entre los habitantes del área rural marginada del estado de Durango, ubicado en la región norte de México. Esta diferencia se podría explicar si se considera que el contexto cultural y socioeconómico, en el que interviene el estrés asociado al estilo de vida, incrementa las cifras de presión arterial, ${ }^{8}$ de este modo, es posible afirmar que la prevalencia de HAS depende, entre otros factores, del grado de 
desarrollo económico ${ }^{8,15, *}$ y de las condiciones de vida relacionadas con la clase social. ${ }^{8}$ Las diferencias entre la población del medio urbano y la del rural se han estudiado en otros países; ${ }^{3}$ se destacan, además de las causas dependientes del contexto socioeconómico, las relacionadas con el medio ambiente y los factores inherentes a la población como son, por ejemplo, el género, la edad y el consumo de alcohol. Esta interacción de subsistemas personales, sociales y ecológicos determina una compleja relación en la que, para una adecuada comprensión de la frecuencia y las causas relacionadas con la hipertensión, es necesario considerar en forma integral los diferentes subsistemas involucrados, objetivo que va más allá de los alcances de este trabajo. La prevalencia de HAS en las comunidades de menos de 250 habitantes fue de $14.74 \%$, lo cual indirectamente refleja la participación de diferentes factores y condiciones relacionados con los subsistemas sociales, ecológicos y personales que definen a cada población y que, al parecer, determinan una relación directa entre la prevalencia de hipertensión y el grado de desarrollo de la comunidad.

La prevalencia de HAS que notificaron Vázquez y colaboradores, ${ }^{15}$ para la zona rural de Jalisco a principios de la década de los ochenta, fue de 7.7 y $10.8 \%$, mientras que en este trabajo resultó de $19.26 \%$ para los hombres y de $22.71 \%$ para las mujeres. Esas diferencias podrían atribuirse al tamaño de la muestra, a la metodología que se aplicó, y a que entre uno y otro estudio transcurrieron cerca de dos décadas. En ese contexto, en el área urbana del país la prevalencia de HAS se incrementó en $2 \%$ de 1991 a 1993, ${ }^{13,14}$ por lo que podrían esperarse crecimientos similares en la frecuencia de la enfermedad en otros sectores de la población como el rural.

En el área urbana la distribución de la hipertensión, según el género, es discretamente superior en los hombres, con una razón hombre/mujer de 1.1:1; en la zona rural marginada, se documentó una relación inversa, con un discreto predominio de la HAS en la mujer (razón mujer/hombre de 1.18:1) que se incrementó en las comunidades rurales de menos de 250 habitantes (razón mujer/hombre de 1.23:1). Este hallazgo ya lo habían notificado Vázquez y colaboradores, ${ }^{15}$ quienes encontraron que la prevalencia de

\footnotetext{
* Saunders N. Trabajo presentado en el VIII Congreso Mundial de Cardiología 1978 sept; Tokio, Japón. Citado por: González-Caamaño A, Pérez-Balmes J, Nieto-Sánchez C, Vázquez-Contreras E. Importancia de las enfermedades cronicodegenerativas dentro del panorama epidemiológico actual de México. Salud Publica Mex 1986;28:8.
}

hipertensión arterial en las mujeres de la zona rural es $3.1 \%$ más elevada que la de los hombres, cifra similar a la encontrada en este estudio (3.4\%) y que pudiera expresar la presencia de problemas derivados de la técnica de muestreo, o bien, diferencias en la estructura poblacional de la zona rural en comparación con la urbana. En ese contexto, la mayor participación femenina observada en el área rural marginada del país podría ser consecuencia del fenómeno migratorio que involucra, sobre todo, a los hombres jóvenes -pues se estima que aproximadamente $80 \%$ de la población rural activa es trabajador migrante $-{ }^{17} \mathrm{y}$ de que, en general, la mujer permanece durante más tiempo en su lugar de origen, a diferencia del hombre, ${ }^{19}$ así entonces, más que un sesgo de selección, la mayor proporción de mujeres podría ser representativa de la estructura poblacional del área rural marginada de México, ${ }^{17}$ cuyas características son diferentes a las de la población general del área urbana. En el mismo contexto, en los sujetos mayores de 50 años de la población estudiada se encontró una prevalencia de hipertensión superior a $25 \%$, aunque inferior, no obstante, a la observada en el área urbana, ${ }^{14}$ en estos resultados podría influir la redistribución de la estructura por edad y género que se observa en las comunidades rurales. ${ }^{18}$

Según el informe de la ENEC, ${ }_{1}^{14}$ la razón diagnóstico previo/hallazgo de casos con hipertensión arterial es de 1:1.3; es decir, por cada paciente que se sabía hipertenso se identificaron 1.3 casos de nuevo diagnóstico. $\mathrm{Al}$ respecto dicha razón fue notoriamente inferior (0.44), lo que puede representar una tasa de crecimiento de la enfermedad diferente a la documentada en la zona urbana, $\mathrm{y} / \mathrm{o}$ probablemente se relacione con la menor participación de hombres, respecto a los que se ha documentado una tasa de incidencia de hipertensión tres veces mayor que en las mujeres..$^{12} \mathrm{En}$ este estudio el hallazgo de hipertensión de nuevo diagnóstico fue mayor en los hombres, fenómeno que puede relacionarse con el hecho de que los varones acuden a la consulta médica con menor frecuencia y, en tal medida, disminuyen sus posibilidades de un diagnóstico temprano de HAS.

Los factores de riesgo para hipertensión identificados en el análisis multivariado fueron la obesidad, el diagnóstico de diabetes tipo 2 y el consumo de tabaco y alcohol. En el norte de México, el hábito tabáquico está presente en $28.3 \%$ de la población urbana con $\mathrm{HAS}^{14}$ mientras que en la población rural marginada se identificó en $53.8 \%$ de los pacientes hipertensos, lo cual pone de manifiesto la elevada exposición a uno de los principales factores de riesgo asociados a problemas cardiovasculares. ${ }^{2,15}$ 
La obesidad, la hipertensión arterial y la diabetes tipo 2 son las enfermedades crónicas de mayor prevalencia, pues entre 70 y $80 \%$ de los sujetos adultos padecen, por lo menos, una de estas tres enfermedades; de hecho, los estudios epidemiológicos muestran una clara superposición entre HAS-obesidad-DMNID. ${ }^{20}$ De las variables asociadas con la hipertensión arterial, la obesidad es una de las más importantes, ${ }^{2,20-22}$ su prevalencia en el área urbana fluctúa entre 55 y $63 \%$ en las mujeres y entre 28 y $50 \%$ en los hombres. ${ }^{21}$ Por el contrario, en la población rural bajo estudio la frecuencia de obesidad es muy baja ( $9.4 \%$ de la población total), y se distribuye en $4.9 \%$ en los sujetos sin HAS y $25.2 \%$ de los sujetos hipertensos, lo que probablemente se relaciona con las características del hábito dietético y/o una mayor actividad física, que son diferentes a las observadas en comunidades urbanas.

La prevalencia de diabetes tipo 2 entre los sujetos hipertensos fue más elevada que en los no hipertensos, hallazgo similar al descrito por Formiguera y colaboradores,$^{20}$ y que expresa de manera indirecta la compleja relación obesidad-DMNID-HAS, en la que la resistencia a la insulina parece ser el factor común.

Otro de los factores asociados a HAS es el consumo de alcohol,; la ingesta de 24-30 g alcohol/día o más se asocia con elevación de la presión arterial, y en los sujetos que consumen 48-60 g alcohol/día, la presión arterial diastólica se eleva de 2 a $4 \mathrm{mmHg}{ }^{23} \mathrm{En}$ la población de este estudio, $28.5 \%$ de los sujetos con hipertensión arterial consumían $50 \mathrm{~g}$ alcohol/ día o más, en comparación con $7.4 \%$ de los no hipertensos, lo que aporta evidencias sobre esta asociación.

Finalmente, cabe destacar que aproximadamente la mitad de los pacientes con HAS tuvo antecedente familiar de hipertensión, cifra que pudiera subestimar su verdadera frecuencia, ya que el antecedente de hipertensión es ignorado por una elevada proporción de individuos. ${ }^{7} \mathrm{Al}$ respecto, llama la atención el hallazgo de la elevada frecuencia, en los sujetos con HAS, del antecedente familiar de hipertensión en la rama materna; no se tienen informes previos para comparar estos resultados, por lo que será necesario realizar estudios específicos para establecer las características del patrón de transmisión genética en la hipertensión arterial.

\section{A gradecimientos}

Se agradece el apoyo de los doctores Juan Martínez Arias y Francisco Sandoval Herrera, coordinadores delegacionales del programa IMSS-Solidaridad en Durango, así como la participación entusiasta y decidida de los doctores Joel Mejorado Bretado, José Guadalupe Castañeda López, Higinio Caballero Barraza, Rafael Moreno Gallegos, Luis Torres Guereca, Oscar Ureta Quintero, Gerardo Arellano del Toro y Alfredo Martínez Martínez, asesores del Programa IMSS-Solidaridad. Asimismo se agradece el decidido apoyo de todo el personal médico adscrito a dicho programa, de las autoridades delegacionales y de Fidel Solís, quien capturó los datos recabados. Sin su colaboración no hubiera sido posible el llevar a término este proyecto.

\section{Referencias}

1. González-C aamaño A, Pérez-Balmes J, N ieto-Sánchez C, Vázquez-C ontreras $\mathrm{E}$. Importancia de las enfermedades cronicodegenerativas dentro del panorama epidemiológico actual de México. Salud Publica Mex 1986; 28:3-13.

2. Chávez-D omínguez R, Vega-Estens $P$, Larios-Saldaña MA. La hipertensión arterial y otros factores de riesgo coronario en la atención del primer nivel. Arch Inst C ardiol Mex 1993;63:425-434.

3. García-De Alba JE, A rias ME, Parra-Carrillo JZ,Velazco-López T. Algunos aspectos de la presión arterial en trabajadores urbanos. Arch Inst Cardiol Mex 1990;60:89-98.

4. N oa-O rtega FH, Castillo-Herrera J, Heredero-Baute L, Castro-García A, Bacallao-Gallestey J, Rojas-Ramos M. Factores genéticos y ambientales en pacientes con hipertensión arterial esencial en una población urbana de Cuba. Arch Inst Cardiol Mex 1995;65:426-434.

5. C hávez-D omínguez R. La hipertensión arterial y el procedimiento en las encuestas. Estudios de población encauzados al control del padecimiento. Arch Inst Cardiol Mex 1986;56:453-458.

6. Chávez-C arbajal JF, Herrera JE, Salazar LA, Vidal J, Moreno A, Tena Y et al.Valores de la presión arterial en diversos grupos de población urbana de la ciudad de Morelia. Arch Inst C ardiol Mex 1990;60:577-586.

7. Kornhauser C, Malacara JM, D onato F, Botello D. Mayor prevalencia de hipertensión arterial en mujeres mayores de 50 años en la ciudad de León, Guanajuato. Rev Invest C lin 1994;46:287-294.

8. D ressler W, Grell G, Gallagher PN, Viteri F. Blood pressure and social class in a Jamaican community. Am J Public Health 1988;78:714-716.

9. G onzález-C aamaño A. Epidemiología de la hipertensión arterial en México: estudio en el Municipio de Toluca. México, D.F.: Consejo Mexicano contra la Hipertensión Arterial/Ediciones Médicas Actualizadas, 1982.

10. Gonzalez-Caamaño A, Cooper R.A blood pressure survey in $\mathrm{N}$ uevo Laredo, Mexico. Public Health Rep 1982;97:116-121.

11. Piña B. Prevalencia de hipertensión arterial en un grupo de derechohabientes del Instituto Mexicano del Seguro Social (IMSS). Rev Med IMSS 1981;19:217-221.

12. Escamilla-C ejudo JA, López-Cervantes M, Esco bedo-De la Peña J, Bustamante-Montes P. Prevalencia de hipertensión arterial y factores asociados en una delegación política de la ciudad de México. Arch Inst $C$ ardiol Mex 1992;62:267-275.

13. Tapia-C onyer R. Encuesta N acional de Enfermedades Crónicas. México, D.F.: Secretaría de Salud, 1991:16-19.

14. Tapia-C onyer R. Encuesta N acional de Enfermedades Crónicas. México, D.F.: Secretaría de Salud, 1993:25-28.

15. Vazquez-Valls E, García-De Alba C, Arias-Merino DE, Parra-Carrillo JZ. Algunos aspectos de la presión arterial en el área rural de Jalisco. Salud Publica Mex 1981;23:83-92. 
16. G uerrero-Romero JF, Rodríguez-Morán M, Sandoval-Herrera F. Prevalencia de diabetes mellitus no insulino dependiente en la población rural de Durango, México. Rev Panam Salud Publica 1997;2(6):386-391.

17. Flores-Alvarado A. Características socioeconómicas de la población marginada rural. En: Diagnóstico de Salud en las Zonas Marginadas Rurales de México, 1986-1991. México, D.F.: Instituto Mexicano del Seguro Social, 1992:39-56.

18. Simposio sobre hipertensión en A mérica Latina. Bol 0 ficina Sanit Panam 1988;105:203-208.

19. Sánchez-G irón C, Brust-Mascher E, De Icaza-Herrera M,Vidrio-Amor $H$, Brust-C armona H. Evaluación de la frecuencia cardiaca y presión arterial en habitantes de MilpaAlta, D.F. Salud Publica Mex 1992;34:434-448.
20. Formiguera X, Barbany M. 0 besidad e hipertensión arterial: una complicada relación. Endocrinología 1992;38:389-393.

21. González-Villalpando C, Stern MP. La obesidad como factor de riesgo cardiovascular en México. Estudio en población abierta. Rev Invest Clin 1993;45:13-21.

22. $\mathrm{N}$ ational Institutes of Health Consensus Development Panel on the Health Implications of $O$ besity. Health implications of obesity. Ann Intern Med 1985;103:147-151.

23. Alcohol and hypertension: Implications for prevention and treatment. Ann Intern Med 1986;105:124-126. 